\title{
What Is Life-and When Do We Search for It on Other Worlds
}

\author{
Christopher P. McKay
}

\begin{abstract}
There has been considerable attention on how to detect life on other worlds by searching for biomolecules. However, there has been much less clarity as to when it becomes warranted to focus a mission on the search for life on another world. At a minimum, a life-detection mission should follow convincing evidence of (1) Liquid water of suitable salinity, past or present; (2) Carbon in the water; (3) Biologically available N in the water; (4) Biologically useful energy in the water; (5) Organic material that can possibly be of biological origin and a plausible strategy for sampling this material. Based on these prerequisites, the most promising targets for a life search are currently the plume of Enceladus and the subsurface of Mars-in equatorial lake bed sediments and in polar ice-cemented ground. Neither the surface of Europa nor the clouds of Venus meet the criteria listed here but may with further exploration. Key Words: Life detection-Enceladus-Mars. Astrobiology 20, 163-166.
\end{abstract}

\section{Introduction}

$\mathbf{T}$ HERE IS GROWING INTEREST in life-detection missions to the other worlds of our Solar System. There is a real prospect that the next decade of planetary missions will include missions whose primary objective is the search for life. Preparations for such missions have focused on how to search for evidence of life (McKay, 2004; Hand et al., 2017; Neveu et al., 2018). We need more consideration of which worlds to target. Unfortunately, based on our current knowledge, the list of suitable candidates is quite short, and the list of potentially false leads is longer.

The Viking Mission to Mars, in 1976, was the first, and to date the only, life-detection planetary mission. The Viking approach was to search for microorganisms in the soil that would metabolize when provided with nutrients. This approach requires that the organisms are alive and that their growth conditions are understood in advance. It is now known that many soil microorganisms are undetectable by such methods (e.g., Steven et al., 2007). At the time of Viking, there was virtually no alternative. In contrast, current life-detection approaches are based on the detection of biomolecules such as amino acids and lipids that would show a characteristic pattern if produced by biology (Lovelock, 1965; McKay, 2004; Hand et al., 2017; Neveu et al., 2018). Instruments are in hand to do these tests at the relevant level of detection compared to abiotic sources of these same compounds (Hand et al., 2017).

Of course, these methods assume that life is biochemically similar, but not necessarily identical, to life on Earth.
This is a plausible assumption for life in liquid water environments. A more general approach would be to directly search for Darwinian evolution-the defining property of life and the ultimate criteria that must be met to determine whether a chemical system represents life (e.g., Benner, 2010). An approach for detecting Darwinian evolution on Mars has been proposed (Chao, 2000) but requires a sample that contains living organisms and further that, like the $\mathrm{Vi}$ king biology experiments, the conditions can be made such that these organisms will grow and reproduce. In contrast, the detection of biomolecules can in principle detect the presence of life even if only dead organisms are present in the sample. The pattern and complexity of the biomolecules provide indirect evidence of past selection by Darwinian evolution (Benner, 2010, 2017).

\section{Prerequisites for a Life-Detection Mission}

We have a strategy to detect life, but there has been much less consideration as to when it is justified to search for life on other worlds. Here I propose a set of prerequisites for a life-detection mission, based on well-established requirements for life (e.g., Benner et al., 2004) and the practicalities of doing the relevant analyses on a planetary mission. Given these prerequisites, we can systematically examine the current knowledge of the possible targets in our solar system and determine where a search for life is most likely to succeed, where success is defined as the detection of persuasive evidence of life or a convincing determination that life is not present and in either case reduces significantly the uncertainty

NASA Ames Research Center, Moffett Field, California. 
in the Bayesian priors associated with the question of life on other worlds (Lorenz, 2019).

At a minimum a life-detection mission should follow convincing evidence of

(1) Liquid water of suitable salinity, past or present.

(2) Carbon in the water.

(3) Biologically available $\mathrm{N}$ in the water.

(4) Biologically useful energy in the water.

(5) Organic material that can possibly be of biological origin and a plausible strategy for sampling this material.

Certainly, the motto "follow the water" has been a useful guide in considering astrobiologically interesting targets, but clearly water alone is not sufficient for life. Beyond carbon, the key element on the list above is nitrogen. The rationale for these elements $(\mathrm{H}, \mathrm{O}, \mathrm{C}$, and $\mathrm{N})$ is that together they form $99.5 \%$ of the atoms in microbes (Davies and Koch, 1991), and all have chemical forms that should allow detection. Phosphorous and sulfur, the remaining two of the set known as the biogenic elements, would further strengthen the case for life detection. After $\mathrm{N}$, the next four elements in microbial life (E. coli) are, in order, $\mathrm{P}, \mathrm{Na}, \mathrm{K}$, and $\mathrm{S}$ (Davies and Koch, 1991). On Earth, biologically available energy is either sunlight or chemical redox energy. Prerequisite \#5 is motivated by the fact that obtaining the organic remains of life is required for the biomolecule-based methods proposed (McKay, 2004; Hand et al., 2017).

Any finite list of prerequisites for a life-detection mission can be criticized on the grounds that it does not unequivocally establish habitability. But this is inevitable; habitability is not a precisely defined concept independent of organisms. In the detail, the habitability of an environment is relative to the organism that is inhabiting that environment, and the growth of an organism is ultimately the only definitive proof of habitability. This difficultly in precisely defining habitability is illustrated with an example: there is no environment on Earth that is habitable for all organisms on Earth.

The requirements for the origin of life are not included in the list presented above because these requirements are unknown. There is no clear indication of how the life we see on Earth originated, where on Earth or elsewhere it originated, the essential environmental conditions, or the time required for its origin. The geological and phylogenic records indicate the presence of life on Earth prior to 3.5 billion years ago but provide no other firm constraint on its origin. The supposition that life originated on Earth itself is neither proven nor refuted by available data.

\section{Looking for Life...}

Based on the five prerequisites for a life-detection mission listed above, the most promising target for a life search is the plume of Enceladus. The Cassini mission has shown that the plume derives from an ocean below the ice with salinity comparable to Earth's ocean and in contact with lowtemperature hydrothermal flows. Carbon as organic material and $\mathrm{CO}_{2}$ are present as well as biologically available nitrogen compounds (Waite et al., 2009). Chemical redox energy couples, including $\mathrm{H}_{2}$ and $\mathrm{CO}_{2}$, have been detected (Waite et al., 2017). Organic material present in the plume could be of recent biological origin (Steel et al., 2017) and could be sampled by moving through the plume (Reh et al., 2016). The biggest challenge is collecting enough material for analysis in the thin plume at a low enough velocity that the biomolecules remain intact.

With the detection of nitrates (Stern et al., 2015) and organic compounds (Freissinet et al., 2015; Eigenbrode et al., 2018) in the surface soils of Mars, all the five prerequisites for a life-detection mission listed above are satisfied for past environments on Mars. At the Gale Crater site, near the equator, there is evidence of suitable liquid water in the past (Grotzinger et al., 2015) with sunlight accessible, possibly through a permanent ice cover (Kling et al., 2020). Carbon as $\mathrm{CO}_{2}$ is readily present. In the polar regions investigated by the Phoenix mission (Smith et al., 2009), there is formation of liquid water in the ice-cemented ground at high obliquity in the recent past (McKay et al., 2013), and redox couples based on perchlorate could support life in the subsurface. Thus, on Mars there are two likely targets that satisfy the five prerequisites for a search: equatorial lake sediments and polar ice-cemented ground. In both cases, the biggest challenge is drilling deeply to the subsurface layers that hold the organic record. The ExoMars mission (Vago et al., 2017) will have a drill and instruments capable of detecting biomolecules, thus advancing the search in the equatorial regions of Mars. The Icebreaker mission (McKay et al., 2013), if selected, would also carry a drill advancing the search for biomarkers in the polar regions of Mars.

\section{4. ...in All the Wrong Places}

Europa has been the focus of considerable attention driven by interest initiating outside the science community. The result has been a serious study of a lander mission to search for biomolecules indicative of life in the top few centimeters of the ice surface (Hand et al., 2017). Considering the prerequisites listed above, such a mission is not scientifically supportable. While there is persuasive evidence for liquid water under the ice on Europa, the composition of the liquid is only indirectly determined-although a suitable salinity is highly likely. While this satisfies the first prerequisite on the list above, none of the other four are met based on the data available. There is no evidence for carbon in the ocean nor evidence for biologically available nitrogen. There is no evidence for redox energy couples in the water, and there are arguments that they may be depleted (Gaidos et al., 1999) and only speculative models for how they might be recharged from the atmosphere (Russell et al., 2017). Perhaps most significantly, if there is life in a subsurface ocean, the case for organic remains of that life being accessible on the surface in the top few centimeters is difficult to make. It is certainly possible that further exploration of Europa and the possible plumes (Sparks et al., 2016) will show that all the prerequisites listed above are indeed met on Europa, but currently this is not that case: Europa is not yet a promising target.

The clouds of Venus have also received attention lately as a possible target for life detection (Limaye et al., 2018), although no detailed missions are under consideration. While the clouds of Venus are composed of sulfuric acid, it is known that microbial life-forms on Earth can survive in such 
acidic conditions. Carbon as $\mathrm{CO}_{2}$, nitrogen as $\mathrm{N}_{2}$, and energy as sunlight complete most of the prerequisites for the cloud environment. Indeed, the only prerequisite lacking for the clouds of Venus is the detection of organic material that can plausibly be the remains of biology. Because the cloud environment is detached from the surface materials, there might be a higher emphasis placed on the detection of the nonvolatile elements needed for life $(\mathrm{P}, \mathrm{Na}, \mathrm{K}, \mathrm{S})$ in preparation for a life-detection mission. Although Venus is not a promising target by the criteria established above, it comes close.

Other possible targets suggested for a life search include the clouds of Jupiter (Sagan and Salpeter, 1976), Ceres (CastilloRogez et al., 2020), Vesta (Houtkooper, 2011), Triton, Pluto, and even the Moon (Schulze-Makuch and Crawford, 2018). None meet the five requirements listed.

\section{Now for Something Completely Different}

As a possible target for life search, Titan is in a class of its own. One could make the case that, if it is possible to substitute liquid methane mixed with ethane in place of liquid water (e.g., Benner et al., 2004) in the first prerequisite, then Titan meets all five. However, that substitution essentially invalidates the entire list. The list is based on life as we know it on Earth. If there is life in the hydrocarbon liquids on Titan, that life is certainly not in any way related to life as we know it. The search for life on Titan must follow a more general approach without presumption of the organic details of life that might be there. McKay (2016) suggested monitoring $\mathrm{H}_{2}$ near the surface and searching through organics at the surface for unexpected patterns, including homochirality. The Dragonfly mission, recently selected, will make important steps in these directions when it arrives at Titan in the mid 2030s.

\section{Conclusion}

Based on our current knowledge of the other worlds in the Solar System, I have argued that only Mars and Enceladus are promising targets for a search for evidence of life. The clouds of Venus almost meet all criteria for a life-detection mission with the important exception of the detection of organic material in the clouds. Further investigation may move many other worlds into the promising categoryEuropa, for example.

However, priorities are not simply set by science. NASA, ESA, and other publicly funded space agencies may have priorities set by a political as well as scientific process. Private organizations, such as the Breakthrough Initiatives, have also contemplated life-detection missions (Worden et al., 2018).

The astrobiology science community should push for life-detection missions to Mars and Enceladus now and engage in further exploration of the interesting worlds that are not promising targets to see whether they can be moved into the list.

\section{References}

Benner, S.A. (2010) Defining life. Astrobiology 10:1021-1030. Benner, S.A. (2017) Detecting Darwinism from molecules in the Enceladus plumes, Jupiter's moons, and other planetary water lagoons. Astrobiology 17:840-851.
Benner, S.A., Ricardo, A., and Carrigan, M.A. (2004) Is there a common chemical model for life in the Universe? Curr Opin Chem Biol 8:672-689.

Castillo-Rogez, J.C., Neveu, M., Scully, J.E.C., House, C.H., Quick, L.C., Bouquet, A., Miller, K., Bland, M., De Sanctis, M.C., Ermakov, A., Hendrix, A.R., Prettyman, T.H., Raymond, C.A., Russell, C.T., Sherwood, B.E., and Young, E. (2020) Ceres: astrobiological target and possible ocean world. Astrobiology 20, doi:10.1089/ast.2018.1999.

Chao, L. (2000) The meaning of life. BioScience 50:245-250.

Davies, R.E. and Koch, R.H. (1991) All the observed Universe has contributed to life. Philos Trans R Soc Lond B Biol Sci 334:391-403.

Eigenbrode, J.L., Summons, R.E., Steele, A., Freissinet, C., Millan, M., Navarro-González, R., Sutter, B., McAdam, A.C., Franz, H.B., Glavin, D.P., and Archer, P.D. (2018) Organic matter preserved in 3-billion-year-old mudstones at Gale Crater, Mars. Science 360:1096-1101.

Freissinet, C., Glavin, D.P., Mahaffy, P.R., Miller, K.E., Eigenbrode, J.L., Summons, R.E., Brunner, A.E., Buch, A., Szopa, C., Archer, P.D., Jr., and Franz, H.B. (2015) Organic molecules in the Sheepbed mudstone, Gale Crater, Mars. $J$ Geophys Res Planets 120:495-514.

Gaidos, E.J., Nealson, K.H., and Kirschvink, J.L. (1999) Life in ice-covered oceans. Science 284:1631-1633.

Grotzinger, J.P., Gupta, S., Malin, M.C., Rubin, D.M., Schieber, J., Siebach, K., Sumner, D.Y., Stack, K.M., Vasavada, A.R., Arvidson, R.E., and Calef, F. (2015) Deposition, exhumation, and paleoclimate of an ancient lake deposit, Gale Crater, Mars. Science 350, doi:10.1126/science.aac7575.

Hand, K.P., Murray, A.E., Garvin, J.B., Brinckerhoff, W.B., Christner, B.C., Edgett, K.S., Ehlmann, B.L., German, C.R., Hayes, A.G., Hoehler, T.M., Horst, S.M., Lunine, J.I., Nealson, K.H., Paranicas, C., Schmidt, B.E., Smith, D.E., Rhoden, A.R., Russell, M.J., Templeton, A.S., Willis, P.A., Yingst, R.A., Phillips, C.B., Cable, M.L., Craft, K.L., Hofmann, A.E., Nordheim, T.A., Pappalardo, R.P., and the Project Engineering Team. (2017) Report of the Europa Lander Science Definition Team, JPL D-97667, Jet Propulsion Laboratory, Moffett Field, CA.

Houtkooper, J.M. (2011) Glaciopanspermia: seeding the terrestrial planets with life? Planet Space Sci 59:1107-1111.

Kling, A.M., Haberle, R.M., McKay, C.P., Bristow, T.F., and Rivera-Hernandez, F. (2020) Subsistence of ice-covered lakes during the Hesperian at Gale crater, Mars. Icarus 338:113495, doi: 10.1016/j.icarus.2019.113495

Limaye, S.S., Mogul, R., Smith, D.J., Ansari, A.H., Słowik, G.P., and Vaishampayan, P. (2018) Venus' spectral signatures and the potential for life in the clouds. Astrobiology 18: 1181-1198.

Lorenz, R.D. (2019) Calculating risk and payoff in planetary exploration and life detection missions. Adv Space Res 64: 944-956.

Lovelock, J.E. (1965) A physical basis for life detection experiments. Nature 207:568-570.

McKay, C.P. (2004) What is life-and how do we search for it in other worlds? PLoS Biol 2, doi:10.1371/journal.pbio.0020302.

McKay, C.P. (2016) Titan as the abode of life. Life 6, doi: 10.3390/life6010008.

McKay, C.P., Stoker, C.R., Glass, B.J., Davé, A.I., Davila, A.F., Heldmann, J.L., Marinova, M.M., Fairen, A.G., Quinn, R.C., Zacny, K.A., and Paulsen, G. (2013) The Icebreaker Life mission to Mars: a search for biomolecular evidence for life. Astrobiology 13:334-353. 
Neveu, M., Hays, L.E., Voytek, M.A., New, M.H., and Schulte, M.D. (2018) The ladder of life detection. Astrobiology 18: 1375-1402.

Reh, K., Spilker, L., Lunine, J.I., Waite, J.H., Cable, M.L., Postberg, F., and Clark, K. (2016) Enceladus Life Finder: the search for life in a habitable Moon. In 2016 IEEE Aerospace Conference, IEEE, Piscataway, NJ, doi:10.1109/AERO.2016 .7500813 .

Russell, M.J., Murray, A.E., and Hand, K.P. (2017) The possible emergence of life and differentiation of a shallow biosphere on irradiated icy worlds: the example of Europa. Astrobiology 17:1265-1273.

Sagan, C. and Salpeter, E.E. (1976) Particles, environments, and possible ecologies in the jovian atmosphere. Astrophys $J$ Suppl Ser 32:737-755.

Schulze-Makuch, D. and Crawford, I.A. (2018) Was there an early habitability window for Earth's Moon? Astrobiology 18 : 985-988.

Smith, P.H., Tamppari, L.K., Arvidson, R.E., Bass, D., Blaney, D., Boynton, W.V., Carswell, A., Catling, D.C., Clark, B.C., Duck, T., and DeJong, E. (2009) $\mathrm{H}_{2} \mathrm{O}$ at the Phoenix landing site. Science 325:58-61.

Sparks, W.B., Hand, K.P., McGrath, M.A., Bergeron, E., Cracraft, M., and Deustua, S.E. (2016) Probing for evidence of plumes on Europa with HST/STIS. Astrophys $J$ 829, doi: 10.3847/0004-637X/829/2/121.

Steel, E.L., Davila, A., and McKay, C.P. (2017) Abiotic and biotic formation of amino acids in the Enceladus ocean. Astrobiology 17:862-875.

Stern, J.C., Sutter, B., Freissinet, C., Navarro-González, R., McKay, C.P., Archer, P.D., Buch, A., Brunner, A.E., Coll, P., Eigenbrode, J.L., and Fairen, A.G. (2015) Evidence for indigenous nitrogen in sedimentary and aeolian deposits from the Curiosity rover investigations at Gale Crater, Mars. Proc Natl Acad Sci USA 112:4245-4250.

Steven, B., Briggs, G., McKay, C.P., Pollard, W.H., Greer, C.W., and Whyte, L.G. (2007) Characterization of the microbial diversity in a permafrost sample from the Canadian High Arctic using culture-dependent and culture-independent methods. FEMS Microbiol Ecol 59:513-523.

Vago, J.L., Westall, F., and the ExoMars Project Team (2017) Habitability on early Mars and the search for biosignatures with the ExoMars Rover. Astrobiology 17:471-510.

Waite, J.H., Lewis, W.S., Magee, B.A., Lunine, J.I., McKinnon, W.B., Glein, C.R., Mousis, O., Young, D.T., Brockwell, T., Westlake, J., and Nguyen, M.J. (2009) Liquid water on Enceladus from observations of ammonia and $40 \mathrm{Ar}$ in the plume. Nature 460:487-490.

Waite, J.H., Glein, C.R., Perryman, R.S., Teolis, B.D., Magee, B.A., Miller, G., Grimes, J., Perry, M.E., Miller, K.E., Bouquet, A., and Lunine, J.I. (2017) Cassini finds molecular hydrogen in the Enceladus plume: evidence for hydrothermal processes. Science 356:155-159.

Worden, S.P., Drew, J., and Klupar, P. (2018) Philanthropic space science: the breakthrough initiatives. New Space 6: 262-268.

Address correspondence to: Christopher P. McKay NASA Ames Research Center Space Science Division 245-3 Moffett Field, CA 94035

E-mail: chris.mckay@nasa.gov

Submitted 9 July 2019

Accepted 23 October 2019 